Questions vives

\section{Questions Vives}

Recherches en éducation

$N^{\circ} 25$ | 2016

L'activité des enseignants face au décrochage scolaire : Quelles difficultés et quelles mises en œuvre professionnelles?

\title{
Façonner une identité enseignante pour ne pas décrocher professionnellement et soutenir la réussite des élèves
}

Shaping a teacher identity in order to avoid dropping the profession and support student success

\section{Philippe Zimmermann}

\section{(2) OpenEdition}

12 Journals

\section{Édition électronique}

URL : http://journals.openedition.org/questionsvives/1898

DOI : $10.4000 /$ questionsvives. 1898

ISSN : $1775-433 \mathrm{X}$

Éditeur

Université Aix-Marseille (AMU)

\section{Édition imprimée}

Date de publication : 7 juillet 2016

ISBN : 978-2-912643-49-0

ISSN : $1635-4079$

\section{Référence électronique}

Philippe Zimmermann, «Façonner une identité enseignante pour ne pas décrocher

professionnellement et soutenir la réussite des élèves », Questions Vives [En ligne], № 25 | 2016, mis en ligne le 05 septembre 2016, consulté le 19 avril 2019. URL : http://journals.openedition.org/

questionsvives/1898; DOI : 10.4000/questionsvives.1898

Ce document a été généré automatiquement le 19 avril 2019.

\section{cc) (†)}

Questions Vives est mis à disposition selon les termes de la licence Creative Commons Attribution -

Pas d'Utilisation Commerciale - Pas de Modification 4.0 International. 


\title{
Façonner une identité enseignante pour ne pas décrocher professionnellement et soutenir la réussite des élèves
}

\author{
Shaping a teacher identity in order to avoid dropping the profession and support \\ student success
}

Philippe Zimmermann

\section{Introduction}

1 Le nombre croissant d'études portant sur le désengagement des enseignants novices, leur retrait, voire sur le décrochage des enseignants (Alava, 2015 ; Hernandez \& Méard, 2014 ; Karsenti, Collin \& Dumouchel, 2013), ou encore sur leurs difficultés d'insertion professionnelle (De Stercke et al., 2010; Mukamurera, Martineau, Bouthiette \& Ndoreraho, 2012) témoigne d'une préoccupation scientifique et institutionnelle grandissante pour l'accompagnement des jeunes enseignants à l'entrée dans le métier. Si le départ prématuré d'enseignants au cours de leurs premières années d'exercice du métier est particulièrement développé outre-Atlantique, en France, son ampleur reste difficile à appréhender même si le phénomène semble être en constante évolution. En évoquant l'idée d'un " double décrochage », Karsenti et Collin (2009) rejoignent plusieurs auteurs qui posent l'hypothèse d'une corrélation entre le décrochage professionnel de l'enseignant et le décrochage scolaire de l'élève (Hugon, 2010 ; Moussay, 2014).

Du côté de l'enseignant, la littérature foisonne depuis plus de 40 ans de recherches pointant, comme le décrivait Lortie dès 1975 , le " choc de l'entrée dans le métier ». Ce constat reste criant d'actualité lorsqu'on observe l'inconfort vécu par les enseignants novices face à la complexité du métier et son évolution permanente (Ria, 2009), les tensions et les doutes qu'ils manifestent face à un métier plus exigeant qu'ils ne l'avaient 
imaginé (Giust-Desprairies, 1996), ou encore leur faible résilience lorsqu'ils manquent d'efficacité et qu'ils perçoivent un manque d'effets de leur pratique sur les apprentissages des élèves (Gu \& Day, 2007 ; Saujat, 2007). Le sentiment d'« incompétence pédagogique » (Martineau \& Presseau, 2003) qu'ils peuvent nourrir au vu de leurs premières expériences en classe, peut expliquer leur difficulté à façonner leur identité professionnelle (Beijaard, Verloop \& Vermunt, 2000) considérée comme un processus en constante évolution (Zimmermann \& Méard, 2015). En effet, face aux contraintes et aux prescriptions sans cesse renouvelées du métier, ils sont constamment amenés à opérer des compromis et à ajuster leurs manières de faire, notamment pour s'impliquer dans le «millefeuille de dispositifs » destinés à endiguer la difficulté scolaire (Felix, Saujat \& Combes, 2012). La réorganisation de leur travail les amène ainsi à reconsidérer leur perception du métier.

Du côté de l'élève, le décrochage scolaire est défini comme la sortie prématurée d'un élève du système éducatif sans diplôme ni qualification. Il est source de nombreux travaux récents (Blaya, 2010 ; Flavier \& Moussay, 2015), notamment en raison d'une prise de conscience politique du lien entre ce décrochage des élèves et des problèmes sociaux qu'il engendre (montée du chômage, précarité professionnelle...) (Janosz, 2000 ; Millet \& Thin, 2005). Cette définition est toutefois inopérante dans le premier degré, où les recherches évoquent davantage des enfants «à risque de décrochage " (Feyfant, 2012) ou des élèves présentant des difficultés scolaires (Bautier et al., 2002). Selon ces auteurs, la genèse $d u$ décrochage scolaire peut être étudiée dès l'école primaire à travers les interactions entre les élèves et l'institution scolaire, les difficultés scolaires étant considérées comme le phénomène le plus facilement repérable d'un processus complexe. Dès lors, la prévention des risques de décrochage scolaire passe par le repérage du manque de réussite scolaire. Le décrochage scolaire, processus difficilement perceptible à son origine, résulterait quant à lui d'un faisceau de facteurs familiaux, socioéconomiques, personnels ou dépendant de l'environnement scolaire, susceptibles de conduire à des stratégies d'évitement, puis au désengagement progressif du travail scolaire (Blaya, 2010; Fortin, Marcotte, Diallo, Potvin \& Royer, 2013; Lee \& Burkam, 2003). D'autres travaux l'envisagent dans la dynamique des interactions avec les enseignants eux-mêmes (Hugon, 2010; Méard, 2013), en développant d'une part l'hypothèse d'un déficit de coconstruction de sens des situations scolaires au cours des interactions entre l'enseignant et ses élèves, d'autre part, celle d'un déficit d'efficience de l'enseignant et des élèves dans les tâches scolaires. Ce double déficit serait à l'origine des difficultés d'accrochage des élèves et des enseignants. Au-delà de l'intérêt indéniable de ces études, l'approche identitaire retenue semble intéressante en ce sens qu'elle se situe au carrefour d'une détermination sociale et d'un investissement individuel et subjectif: on devient enseignant en interaction avec la tutelle, les pairs, les usagers, mais aussi en étant « acteur », en s'investissant personnellement.

Cette étude qualitative développera une analyse in situ des situations de classe, afin de mieux comprendre les tensions identitaires rencontrées par les enseignants novices face à des élèves potentiellement décrocheurs. Trois points de résultats sont développés : l'importance du collectif dans un processus identitaire complexe quand l'enseignant doit faire face à des réactions d'élèves inédites, la reconnaissance mutuelle enseignant/élève, source d'accrochage, et les effets contrastés de l'alternance dans la formation des enseignants. La discussion porte sur l'hypothèse d'un lien spiralaire entre les processus d'accrochage/décrochage professionnel et la réussite des élèves. 


\section{Cadre théorique}

5 Afin d'appréhender le plus précisément possible l'activité de l'enseignant et en accordant, pour y parvenir, le primat à l'acteur, nous nous appuierons sur les postulats de la théorie historico-culturelle de l'activité développée par Vygotski $(1978$; 2003) et Leontiev (1976). Ce dernier définit l'activité comme étant une structure à trois étages : les buts - l'action de l'enseignant, les motifs - ses préoccupations, ce qui le pousse à agir, et les opérations ses manières de faire. Le développement de l'activité des professionnels (dans notre étude, les enseignants novices) est alors considéré comme étant «bi-phasé » (Saujat, 2010 ; Zimmermann, Méard \& Flavier, 2012), c'est-à-dire envisagé à la fois par le « sens » de l'activité - le dépassement des motifs initiaux en lien avec l'évolution des buts, et par l'« efficience» - la mise en œuvre de nouvelles opérations pour atteindre les buts (Leontiev, 1976). Dans cette approche, la situation sociale est considérée comme source et ressource de l'activité individuelle. Le développement du sujet est ainsi compris à travers sa participation à des pratiques socialement organisées, et résulte de l'appropriation de signes culturels externes, transmis en situation dissymétrique (par exemple d'un enseignant chevronné à un enseignant novice), et d'une intériorisation de ces signes qui sont " greffés ", permettant de gagner en autonomie.

6 La réflexion s'inscrit également dans l'approche de la clinique de l'activité (Clot, 1999; 2008), qui prolonge les travaux de Vygotski. L'activité est considérée comme « une infime part de ce qui est réalisable» (Vygotski, 1994), et son analyse nécessite de prendre en compte ce que l'individu aurait souhaité faire, ce qu'il n'a pas pu faire, ce qu'il a été empêché de faire (Clot, 2008). Les activités suspendues, contrariées ou empêchées, celles qui n'ont pas vaincu, continuent à infléchir l'activité du sujet. Elles constituent « une influence contre laquelle il peut rester sans défense » (Clot, 1999, p. 119), ou, au contraire, face à laquelle il peut répliquer, se défendre, surcompenser (Vygotski, 2003), ce qui conduit au développement de son activité. En ce qui concerne les enseignants, cette défense s'opère souvent par l'intermédiaire du collectif de travail (le fonctionnement de l'équipe pédagogique, les gestes professionnels partagés) et leur permet de gagner en efficience. Leur développement professionnel passe également par la création de leur propre «style» au sein d'un «genre» (Clot, 2008) enseignant, qui nécessite de «renormaliser» les prescriptions de la formation initiale (Leblanc, Ria, Dieumegard, Serres \& Durand, 2008) et de métier (Ria, 2009), en s'en affranchissant par la mise en œuvre de nouvelles opérations. Ce cadre nous permettra ainsi de comprendre pourquoi les enseignants parviennent à augmenter leur " pouvoir d'agir ", c'est-à-dire à poursuivre de nouveaux motifs et/ou à mettre en œuvre des opérations inédites pour atteindre de nouveaux buts. Le développement professionnel passe également par la réalisation de compromis entre les contraintes et les prescriptions du métier (Daniellou, 2002 ; Saujat, 2004), dont le caractère multiple et souvent contradictoire est à l'origine de conflits chez les enseignants, qui hésitent à suivre les prescriptions institutionnelles, celles du centre universitaire (le prescripteur secondaire, selon Goigoux, 2002) ou celles des élèves euxmêmes.

7 La perspective identitaire que nous envisageons pose l'hypothèse d'un lien étroit entre l'accrochage des enseignants au métier (leur engagement, leur participation aux dispositifs collectifs, le sens qu'ils accordent à leurs tâches, l'efficience à laquelle ils parviennent), et l'accrochage des élèves au travail scolaire (leur investissement, les motifs 
scolaires qu'ils poursuivent, leur réussite). Ce lien évolue selon un processus dynamique, non linéaire (Zimmermann \& Méard, 2015) et n'est jamais acquis une fois pour toutes ; il reste constamment à construire avec les élèves, ce qui explique les fluctuations de l'identité professionnelle des enseignants novices, se percevant tantôt "enseignant ", " enseignant en devenir » voire " étudiant ». Le processus identitaire est ainsi fonction de la maitrise des opérations par les enseignants (pour tenir la classe, faire apprendre aux élèves, ...), de leur capacité à renormaliser les prescriptions (ajuster son enseignement aux programmes, prescription institutionnelle, ou s'autoriser à s'en affranchir au regard des spécificités des élèves...) et de la reconnaissance (Gernet \& Dejours, 2009) de l'utilité, de la conformité et de l'originalité de leur activité accordée par les pairs, le collectif, les supérieurs hiérarchiques et par les élèves eux-mêmes.

\section{Méthodologie}

\subsection{Contexte et participants}

8 Nous avons travaillé durant deux années consécutives avec dix enseignants novices volontaires du premier degré, issus de deux cohortes, âgés de 21 à 28 ans. Tous avaient été lauréats du concours de recrutement de l'Éducation Nationale et étaient en dernière année de formation initiale à l'École Supérieure du Professorat et de l'Éducation. De plus, tous avaient effectué au cours de leurs études au moins deux stages d'observation courts (une semaine). Quatre d'entre eux avaient déjà validé un master et se trouvaient en situation de passer un diplôme universitaire. À l'instar des six autres participants, ils suivaient une formation alternant des cours dispensés en centre universitaire et un stage en responsabilité de deux jours par semaine, à l'année. Les stagiaires étaient affectés en classe de petite section de maternelle (des élèves âgés de trois ans - Amel, Eve, Gil), de moyenne section (élèves de quatre ans - Mina), de grande section - cours préparatoire (élèves de cinq et six ans - Sam) et de cours moyen (élèves de neuf et dix ans - Ani, Ann, Sofi, Tom, Toni). C'est à l'issue de cette dernière année de formation qu'était décidée leur titularisation, leur prorogation de leur formation d'une année supplémentaire, ou leur licenciement, au regard d'évaluations effectuées en classe au cours de leur stage et en centre universitaire.

\subsection{Recueil des données}

9 Dans un premier temps, nous avons recueilli des traces de l'activité des participants (Clot, 2008), en nous appuyant sur des enregistrements audio-visuels (24 prises de vue en classe) et des carnets de bord. Dans ce « journal intime » individuel, chaque participant renseignait tout événement qui semblait, à ses yeux, constituer une aide à devenir enseignant, ou au contraire, qui ne favorisait pas son devenir enseignant. Ces traces d'activité ont servi de support à 24 entretiens d'auto-confrontation simples et trois entretiens d'auto-confrontation croisés (Clot, 2008), réalisés avec chaque participant en centre universitaire. En cohérence avec notre cadre théorique, nous avons ainsi mis en œuvre une méthodologie indirecte (Yvon \& Clot, 2003) de recueil des données, et avons, au cours des entretiens, laissé le primat - mais non l'exclusivité - aux participants ; nous avons ainsi systématiquement débuté les entretiens par les événements sur lesquels souhaitent revenir les participants, et avons également abordé d'autres événements 
enregistrés ou notés dans les carnets de bord, qui nous semblaient intéressants dans le processus identitaire de l'enseignant.

\subsection{Traitement des données}

10 Le traitement des données s'est déroulé en quatre étapes. a) Dans un premier temps, les entretiens d'auto-confrontation ont été retranscrits verbatim. b) Nous avons alors repéré les motifs, buts et opérations des acteurs afin de formaliser leur activité et les moments où se manifestaient des indicateurs d'accrochage/décrochage, identifiés au cours des entretiens à travers les marques de poursuite/d'arrêt de leur activité. Par exemple, tel enseignant qui exprime qu'il ne servait à rien de continuer sa leçon et qui décide de tout arrêter, ou au contraire, tel autre qui malgré ses difficultés et le fait de se sentir démuni, surpasse l'événement et considère qu'il a appris à mieux gérer la situation... Nous avons formalisé les raisonnements des acteurs sous la forme suivante: [Pour] motif (verbe à l'infinitif), [faire en sorte de] but (verbe à l'infinitif) [en] opération (participe présent). Exemple : [Pour] retrouver le calme en classe (motif), [faire en sorte de] capter l'attention des élèves (but), [en] rejoignant son bureau (opération 1), [en] s'asseyant à son bureau (opération 2), [en] ne disant plus rien (opération 3). c) Les variations de motifs et d'opérations des enseignants novices ont été identifiées systématiquement dans les situations et des récurrences ont été recherchées. d) Le travail a été mené à deux chercheurs, qui ont comparé leurs analyses dans un souci de validation des résultats obtenus. Les points de désaccord ont été discutés en vue d'obtenir un consensus. En cas de désaccord persistant, les éléments du corpus concernés ont été rejetés.

\section{Résultats}

L'ensemble des résultats a permis d'identifier et de présenter dans cette étude trois circonstances influençant le processus d'accrochage/décrochage professionnel: l'importance du collectif, celle d'une reconnaissance mutuelle entre l'enseignant et les élèves, et les effets contrastés de l'alternance. Nous montrerons comment chacune de ces circonstances contribue au façonnage de l'identité professionnelle des participants.

\subsection{Le collectif comme aide à ne pas décrocher}

$12 \mathrm{Au}$ cours des entretiens, l'ensemble des participants pointe les difficultés rencontrées face aux élèves, au point d'« avoir la tête sous l'eau » (Ani), et de se sentir « démunie » (Amel, Mina), «perdue » (Eve), « désarmé » (Toni), « complètement impuissante » (Sofi), voire d'être dans une "période de survie » (Eve) qui se prolonge au-delà des premiers mois. Les jeunes enseignants s'accordent sur le fait de manquer d'opérations pour tenir la classe ou répondre aux élèves. Cela est d'autant plus vrai face à des élèves qui, selon les stagiaires, sont susceptibles de décrocher. Les jeunes enseignants ont en effet une connaissance lacunaire du décrochage et ne se reconnaissent pas dans ces élèves, puisqu'eux-mêmes, à l'inverse, sont dans un processus scolaire et universitaire de réussite. De plus, les motifs de ces élèves sont parfois très éloignés des motifs professionnels de l'enseignant (susciter la motivation, capter l'attention, donner du sens,...) : par exemple, Sofi, préoccupée par l'apprentissage des élèves, note qu' «ils ne pensent qu'à s'amuser, à échanger avec [leurs] camarades ». Ainsi, l'absence de co-construction de sens, cumulée au déficit d'opérations 
des stagiaires pour être efficients, amène les jeunes enseignants à se tourner vers le collectif.

La recherche de moments d'échanges informels est en effet soulignée de manière récurrente par les enseignants novices. Alors que plusieurs d'entre eux pourraient rentrer chez eux lors de la pause méridienne, ils décident aussi souvent que possible de rester à l'école « pour poser plein de questions » (Sam), « pour avoir ces discussions informelles, pour créer du lien avec les collègues» et "parce qu'on apprend plein de choses» (Amel). Les échanges portent moins sur les préparations de séances ou de progressions, que sur la " posture et [l]a pratique » de l'enseignant, la gestion de la classe («je leur raconte, en salle des profs... alors ils me demandent, c'était comment le premier round", Tom) ou d'un élève en particulier («j'ai entendu lorsqu'elles [les collègues chevronnées de l'école] ont été confrontées à un élève autiste, comment elles réagissent ", Amel). Ce partage d'expérience est source d'accrochage en ce sens qu'il permet de prendre conscience de difficultés non résolues même par des personnes expérimentées: «elles m'ont bien fait comprendre que parfois elles se sentaient désarmées, (...) qu'elles étaient impuissantes. Je me rends compte que même avec des années d'expérience, on est parfois mis en difficulté » (Amel). Toutefois, pour d'autres stagiaires, les échanges avec le collectif de l'école sont plus rares. Par exemple, Gil constate que "c'est difficile, elles sont toutes plus âgées que moi, on est que six classes et on n'a pas vraiment le temps de discuter ». Le stagiaire s'en remet alors aux réseaux sociaux pour solliciter un collectif absent dans son établissement, afin, malgré tout, d'«échanger des petites astuces, des idées, (...) partager des conseils » avec d'autres enseignants novices, sur les apprentissages (concevoir un projet dans un domaine spécifique) ou la gestion des comportements (comment s'y prendre face à un élève de trois ans qui pleure).

Nous avons pu observer de manière récurrente une confiance absolue manifestée $a$ priori dans le collectif, et qui s'est traduite pour les jeunes enseignants en la délégation de leur responsabilité à autrui pour enseigner à leur place. Les stagiaires cherchaient pendant ces moments des opérations auprès des collègues. C'est par exemple le cas d'Amel, qui, suite à l'accueil d'élèves de maternelle, se voit proposer par la titulaire de la classe de diriger le moment du regroupement. Mais la stagiaire préfère laisser la titulaire opérer : «c'était le tout début d'année, je me suis dit je vais la laisser faire et ça va être un moyen de pouvoir l'observer, de voir d'autres techniques, des trucs tout bêtes... » (Amel). Dans cette situation, Amel se met volontairement, temporairement en retrait pour pouvoir observer un enseignant chevronné.

Plus étonnant, d'autres s'en remettent à des personnes qui ne sont pas du collectif enseignant. Nous avons observé typiquement cette situation en éducation physique et sportive, lorsque les enseignants novices bénéficient d'un intervenant extérieur. Par exemple à la piscine, la première séance de natation est fréquemment déléguée au maître-nageur. De même, en maternelle, les enseignants novices s'en remettent à l'aide maternelle pour observer ses manières de faire (par exemple pour regrouper les élèves), afin de connaitre les habitudes de la classe. Les jeunes enseignants tentent ainsi de compenser leur manque de connaissance des élèves. Ainsi, Sam a distribué un travail trop difficile aux élèves de maternelle, qui restent inactifs, ne parvenant pas à trouver la solution. Mais c'est à l'aide maternelle, présente dans la classe à ses côtés, que Sam reproche son manque d'intervention: " il fallait qu'elle me prévienne, (...), qu'elle me dise que ça ne marche pas, pour que les élèves ne restent pas une demi-heure à rien faire parce qu'ils n'y arrivent pas ». Les jeunes enseignants s'accordent ainsi à reconnaitre l'aide maternelle comme une ressource essentielle pour la gestion de la classe (selon Amel, « on en a surtout 
besoin pour aider lors des ateliers »). Le maitre-nageur, tout comme l'aide maternelle, ne sont pas des enseignants mais constituent paradoxalement une ressource importante pour combler le déficit d'efficience des enseignants novices, grâce aux gestes de métier qu'ils maîtrisent. C'est parce qu'ils connaissent bien les élèves, ou parce qu'ils sont spécialistes de l'activité, que les jeunes enseignants reproduisent leurs manières de faire. En reprenant à leur compte des opérations d'autrui, ils s'attendent à percevoir un gain immédiat d'efficience et le processus d'accrochage professionnel semble favorisé.

\subsection{Une absence de reconnaissance mutuelle enseignant/élèves source de décrochage}

Le processus d'accrochage professionnel est toutefois plus complexe qu'il n'y parait. En effet, au-delà du besoin d'échanges précédemment décrit, les jeunes enseignants cherchent à voir les documents de préparation de leurs collègues. Ainsi, Eve "picore» partout et reprend tous les documents qu'elle peut trouver, notamment en salle des maitres à la photocopieuse. Elle "calque » les techniques des collègues en reconduisant à l'identique leurs manières de faire; de même, Ann "pioche des documents" dans les classeurs que lui prêtent ses collègues pour l' «aider au niveau des progressions». Or en reprenant à l'identique les opérations et préparations d'autrui, les enseignants novices se rendent compte de l'inefficience de ces manières de faire. Les situations qu'ils décrivent typiquement comme "ne marchant pas» (Amel) s'expliquent par leur manque d'ajustement de la situation aux caractéristiques des élèves. Ils ont donc la perception d'être démunis, notamment face aux élèves aux comportements inattendus ou potentiellement décrocheurs. La situation peut conduire ces jeunes enseignants à remettre en cause les pratiques du collectif voire même leur propre capacité à intégrer le genre enseignant. L'invalidation répétée des pratiques d'autrui, rencontrée particulièrement face à des élèves décrocheurs, les place en effet dans une situation où ils ne peuvent être reconnus par les élèves, et ne se reconnaissent eux-mêmes pas capables de les aider. Cette situation peut conduire au découragement, un investissement moindre, un éloignement des motifs professionnels, et au décrochage.

17 Par ailleurs, il n'est pas rare d'observer les enseignants novices se placer en retrait des élèves, notamment face à des élèves manifestement peu engagés dans le travail et désinvestis. Certains renoncent ainsi à poursuivre la séance face à des élèves " insupportables " (Sam), "agités " (Ani), " déchainés» (Mina), "exécrables», "invivables", "terribles", "insolents » qui "ne se mettent pas au travail» (Sofi). Par exemple, lors d'une séance de sciences avec sa classe de cours moyen deux (CM2), Tom a décidé «de tout arrêter à un moment ", en raison d'une ambiance de classe insuffisamment studieuse. De même, Sofi, au cours d'une séance de mathématiques en cours moyen un (CM1), considère que "ça [ne] servait à rien de continuer " à répéter les consignes face à des élèves inattentifs. Elle s'empêche ainsi d'agir et se dessaisit du problème. À ce moment, le sens du travail de ces enseignants est altéré et leurs préoccupations (maintenir la discipline) s'éloignent de leurs préoccupations premières (enseigner).

18 Nous pointons ici le lien entre la co-construction de sens et le processus de reconnaissance ou d'absence de reconnaissance mutuelle (enseignant - élève). Ancien bon élève, l'enseignant novice ne se reconnait pas dans l'élève décrocheur. Tous deux poursuivent des motifs éloignés. Dans ce cas, il peut reporter la responsabilité sur l'élève (à ce moment, il n'endosse plus ses responsabilités d'enseignant, ce qui conduit à un 
sentiment d'impuissance), ou se décourager et se mettre en retrait en raison de son manque de compétences (il ne se reconnait alors plus comme un professionnel). Réciproquement, l'élève qui se désengage témoigne de l'impuissance de l'enseignant à l'intéresser et à le faire réussir en raison d'opérations mal maîtrisées. Ce processus explique le lien spiralaire entre le décrochage professionnel de l'enseignant et le décrochage scolaire de l'élève.

\subsection{Les effets contrastés des circonstances de l'alternance dans l'accrochage professionnel}

19 L'année de titularisation se déroule selon le modèle de l'alternance. Les données font apparaitre les tensions entre les motifs d'étudiant et ceux d'enseignant. Les jeunes enseignants admettent que les cours en centre universitaire "servent » s'ils sont pratiques et concrets. Par exemple, selon Sam, le fait de pratiquer en éducation physique et sportive lui permet d'utiliser les situations présentées avec les élèves, et les cours illustrés avec des exemples donnent envie d' "être testés tout de suite» en stage. De manière récurrente, les stagiaires soulignent l'importance d'avoir des réponses à des besoins "immédiats", et ont besoin de "concret qu'[ils peuvent] directement mettre en place en classe ». Lorsque la formation ne répond pas à leurs attentes, ils s'organisent pour prendre du recul pendant les cours et effectuer une autre tâche que celle demandée par l'enseignant. Par exemple, Gil « coupe complètement » de son cours parce qu'il n'arrive pas «à le raccrocher à ce qu'[il] vit » en classe, ou Mina " prépare pour [s]a semaine à l'école, [s]es fiches de préparation " parce qu'elle est " débordée par le travail à l'école », parce qu'elle a "du mal à sortir la tête de l'eau » et n'est "pas à jour dans [s] es fiches de préparation». Ce travail effectué en cours permet aux stagiaires de "rattraper [leur] retard"sur leur enseignement. Ainsi, nous avançons qu'en l'absence de «solutions» circonstanciées, ils effectuent une forme de décrochage volontaire lors de formations universitaires pour mieux accrocher en classe. Ainsi, Gil se met «dans [s]a bulle» pour se concentrer sur son travail à mener avec sa classe. Autrement dit, l'urgence des préparations des situations de classe prime sur les formations universitaires, qui apparaissent souvent «utiles pour l'année prochaine, mais pas forcément pour cette année ».

Par ailleurs, les stagiaires considèrent unanimement que les évaluations menées en centre universitaire les obligent à devoir faire "quelque chose de formel ", pour "répondre au système ", "respecter la norme ", et concèdent "chercher toujours la note " (Toni, Tom). C'est également le cas au cours de leur soutenance de mémoire, qui rappelle systématiquement les épreuves orales du concours passé l'année précédente en tant qu'étudiant. Ainsi, pour Sam, "c'était horrible (...). J'étais dans le couloir en train d'attendre toute seule et je me suis revue l'an dernier aux oraux blancs, à attendre aussi dans le couloir et à stresser par rapport à ce que j'allais dire ». De même, selon Tom, la rédaction d'un mémoire n' "est pas le travail d'un enseignant (...). Un prof d'école, normalement, il [ne] rédige pas des mémoires quand il travaille tous les jours ». Tom manifeste son souhait de voir cesser ce processus: "à un moment stop quoi. On a l'impression un peu que c'est une obligation institutionnelle, et qu'on le fait parce que voilà faut bien ». Cet éloignement de motifs professionnels d'enseignants altère le sens que les jeunes enseignants attribuent à leur investissement. La forte reconnaissance institutionnelle accordée au travail universitaire et au mémoire contraste avec une reconnaissance insuffisamment accordée à leur travail en classe. Les enseignants novices sont en effet contraints de s'investir dans des domaines 
éloignés de leurs préoccupations principales (le travail en classe, gérer la discipline, faire apprendre aux élèves), au profit d'un travail perçu comme étant davantage celui d'un étudiant (réussir à valider son année). Certains parviennent à effectuer des compromis qui leur permettent de concilier le travail universitaire et leur enseignement en stage en classe ; d'autres, au contraire, ne parviennent pas à trouver cet équilibre. Ils privilégient alors l'une des deux tâches (les évaluations universitaires ou le travail en classe), s'investissant moins dans l'autre, quitte à décrocher d'un des deux versants de la formation.

21 Le contexte de l'alternance est aussi marqué par des situations en classe, parfois très inconfortables, et qui, paradoxalement, contribuent à l'accrochage professionnel. Les résultats font ainsi apparaître que l'ensemble des stagiaires rencontre des situations « très fortes", qui les "poussent dans [leurs] retranchements à un point de folie» (Mina). Par exemple, Ann est confrontée en cours moyen, à un élève qui apporte à l'école un livre d'éducation sexuelle inapproprié pour de jeunes enfants et qui se vante d'avoir vu des films pornographiques; autre exemple, Mina tente de réconforter un élève âgé de quatre ans contrarié au moment de l'accueil, parce qu'il ne parvenait à obtenir un jouet utilisé par un camarade. Mais ce faisant, l'élève a frappé Mina : «les coups sont partis, claques, poings, pieds... il m'a fortement frappé (...). J'ai gardé la trace de sa main toute la matinée ». Face à ces situations dont ils n'imaginaient même pas qu'elles puissent arriver, ces jeunes enseignants se sentent sur le moment "plus fragiles», "déboussolés ». Mina reconnait avoir mis « un moment à $\left[s^{\prime}\right] e n$ remettre». Toutefois, pour Mina comme pour Ann, la résolution du conflit leur permet de découvrir de nouvelles facettes du métier et les «aide à devenir enseignant » en les confortant dans leur rôle. Grâce au soutien contextualisé de l'équipe éducative qui permet in fine une meilleure connaissance des élèves et du fonctionnement de l'école, les stagiaires se sentent alors "vraiment enseignants " parce qu'ils sont reconnus comme tels par leurs collègues et construisent avec eux les remédiations. Le retour en centre universitaire leur permet de partager leurs expériences avec des formateurs et avec leurs pairs. Le simple fait d'entendre que les autres sont confrontés à des difficultés identiques, "rassure», permet de partager des préoccupations et de ne pas considérer la situation "de manière personnelle " (Ann). Dans ces cas où les comportements des élèves sont très éloignés des attentes scolaires, le retour en centre universitaire favorise l'accrochage professionnel en permettant le partage de préoccupations entre pairs et la mise à distance, par l'analyse réflexive, de l'urgence de la situation.

\section{Discussion}

22 À partir de l'analyse des résultats, trois points de discussion sont abordés afin de comprendre le processus d'accrochage/décrochage professionnel sous une approche identitaire: la nécessité d'être efficient pour ne pas décrocher, en empruntant des opérations au collectif, en se les appropriant, et en partageant ses expériences avec les pairs; la nécessité de renormaliser les prescriptions pour répondre aux exigences du stage en classe; la nécessité d'une reconnaissance réciproque de l'enseignant et des élèves, permettant une co-construction de sens déterminante dans le processus d'accrochage/décrochage professionnel.

23 Les résultats soulignent les efforts importants déployés par les jeunes enseignants pour accrocher le métier d'enseignant. Ils rejoignent les travaux d'Adé, Gal-PetitFaux et Serres (2014) qui pointent la pluralité des ressources convoquées par les enseignants novices, et 
précisent que parmi les ressources recherchées, le collectif de travail est omniprésent. Le collectif est celui qui détient les opérations à mettre en œuvre dans sa classe, les « règles du métier » qui définissent le "genre» (Clot, 2008). Or pris dans l'urgence, les jeunes enseignants adoptent une vision simpliste du métier et reproduisent les opérations d'autrui sans les ajuster aux caractéristiques de leurs élèves. Le décalage qu'ils constatent les conduit à une incompréhension des réponses des élèves. Ils perçoivent alors un manque d'efficience, qu'ils peuvent attribuer aux élèves, à eux-mêmes (leur inefficience est alors à l'origine de l'inefficience des élèves) ou aux collègues. Dans ce dernier cas, les dimensions interpersonnelle et transpersonnelle du métier (Clot, 2008) sont atrophiées, alors même qu'elles constituent une ressource essentielle pour l'engagement des enseignants novices. Dans le processus identitaire, nous avançons que la maîtrise des opérations, sous-tendue par les échanges avec le collectif et l'appropriation du genre enseignant, constitue un levier essentiel (mais pas unique) permettant d'assumer ses responsabilités en classe et d'accrocher au métier. À l'inverse, si le manque d'efficience perdure, il amène à douter du collectif, à poursuivre des motifs éloignés des motifs professionnels, et peut conduire au décrochage professionnel. L'efficacité du rapport au collectif apparait ainsi comme source d'accrochage professionnel. Outre l'importance de la relation dissymétrique (Vygotski, 2003) entre un enseignant novice et un enseignant chevronné pour apprendre les techniques du métier, nous avons montré également la nécessité de la relation " symétrique » des jeunes enseignants entre eux, notamment pour se rassurer et partager des motifs communs.

Au-delà du collectif, nos analyses rendent compte de la nécessité, pour les enseignants novices, de modifier les conditions de l'alternance; alors même qu'ils n'enseignent en classe qu'à mi-temps, ils transforment certains temps de formation universitaire en temps de préparation du stage. Se disant démunis, perdus, les jeunes enseignants " décrochent " volontairement des cours pour se concentrer sur leurs préparations de classe. Ils développent ainsi une forme de surcompensation (Saujat, 2004; Vygotski, 1994) qui leur permet de se créer des « ressources intermédiaires » pour être efficaces « malgré tout » (Clot, 1995), c'est-à-dire malgré le peu de temps dont ils disposent. Ils se créent ainsi des dispositifs «sas» (Bruno, Méard \& Walter, 2013), par exemple lors des cours en centre universitaire, leur permettant de travailler davantage et de rattraper leur retard de préparation. Si l'on se réfère aux typologies des élèves en situation de décrochage scolaire (Bonnery, 2003 ; Hugon, 2010), nous pourrions considérer ces enseignants novices comme étant des «décrocheurs de l'intérieur passifs »: en formation à l'université, quand ils sont eux-mêmes «élèves » (Smith \& Ingersoll, 2004), ils décident, de manière volontaire et réfléchie, de ne pas suivre à la lettre la prescription (suivre les cours, prendre des notes...). Ce faisant, ils effectuent une double «renormalisation» de la prescription (Schwartz, 2012) qui contribue à leur accrochage professionnel et à leur construction identitaire : d'une part en tant qu'étudiant en centre universitaire, pour être à jour dans leurs fiches de préparation afin d'être plus efficient en classe, et d'autre part en classe, pour faire face à la multiplicité et au caractère parfois contradictoire des prescriptions institutionnelles. Ce processus apparaitt toutefois comme étant «à double tranchant » (Rots, Kelchtermans \& Aelterman, 2012) en ce sens qu'il peut également être à l'origine d'un décrochage de la formation universitaire proposée et d'un échec lors de la titularisation. C'est notamment le cas lorsque les enseignants novices ne parviennent pas à concilier les exigences du centre universitaire et les attentes de la classe, ou à concilier la « double temporalité » (Bruno, Méard \& Walter, 2013) à laquelle ils sont confrontés : répondre en classe aux besoins les plus urgents des élèves (temporalité à très court 
terme), et se former pour les années futures, à enseigner à des élèves d'un âge et d'un niveau différent, avec des besoins spécifiques (temporalité à plus long terme). Les compromis qu'ils doivent effectuer entre « ce qu'on leur demande de faire » et ce que «ça leur demande » (Saujat, 2004) se jouent donc à la fois dans chaque contexte de la formation (effectuer des compromis en classe d'une part, et en centre universitaire d'autre part), mais également dans la tension entre les deux contextes (ne pas faire exactement ce qu'on leur demande en tant qu'étudiant en centre universitaire parce que le stage en classe leur demande un investissement trop important).

Le processus d'accrochage analysé avec une approche identitaire nous permet aussi d'éclairer la reconnaissance (Gernet \& Dejours, 2009) de l'enseignant novice. Au-delà des reconnaissances qu'il peut obtenir par l'intermédiaire de ses collègues, de ses proches, voire même de l'inspecteur, l'accrochage au métier paraît surtout façonné par des reconnaissances obtenues en classe, des élèves eux-mêmes. Leur engagement dans le travail et leurs réussites sont considérés comme autant de signes de maîtrise de la classe par les enseignants novices. Cela est d'autant plus vrai que la classe leur a été présentée comme étant une classe difficile. Or les stagiaires sont aussi confrontés à des élèves en difficulté et parfois peu investis. Leur comportement, souvent appréhendé pour la première fois au cours du parcours professionnel des jeunes enseignants, est interprété comme un manque de reconnaissance en lien avec des opérations mal maîtrisées. En l'absence de reconnaissance mutuelle, les jeunes enseignants peuvent se désinvestir et décrocher, notamment lorsqu'ils ne parviennent à se rattacher au "genre » (Clot, 2008) pour trouver de nouvelles opérations plus efficientes. L'analyse rejoint les travaux de Clot (2008) qui soulignent l'importance d'« être du métier» mais aussi d'« avoir du métier ». Cette analyse trouve son prolongement au niveau du sens : tout comme au secondaire, les élèves décrocheurs ne poursuivent pas des motifs scolaires (Méard, 2013), les motifs des élèves à risque de décrochage au primaire sont éloignés de ceux de l'enseignant novice qui ambitionne de faire progresser les élèves. On observe ainsi une absence de coconstruction de sens des élèves avec l'enseignant, susceptible de ralentir le processus identitaire et l'accrochage professionnel. La situation est particulièrement complexe pour les enseignants novices en ce sens qu'il leur faut également être reconnus à travers les évaluations en centre universitaire. Cette situation conduit à la poursuite de motifs d'étudiants (par exemple présenter une image conforme devant un formateur, travailler pour avoir la moyenne lors d'une évaluation) très éloignés des motifs enseignants, susceptible d'entraîner, à terme, une situation de décrochage professionnel. L'étude ici menée permet ainsi de renforcer l'hypothèse du lien entre les processus d'accrochage/ décrochage professionnels de l'enseignant et les difficultés scolaires des élèves, déjà envisagée par Hugon (2010) ou Méard (2013). Elle donne également un nouvel éclairage au décrochage enseignant (Karsenti et al., 2013), en soulignant la complexité d'un processus éminemment instable et singulier, mais caractérisé par des éléments communs à l'ensemble des enseignants novices: le besoin de maitrise des opérations, la prise de responsabilité, la renormalisation de la prescription, la reconnaissance par les élèves (Zimmermann \& Méard, 2015). De ces éléments sont fonction les variations d'engagement des enseignants novices, leur façonnage identitaire et leur accrochage professionnel. 


\section{Perspectives}

Cette étude nous amène à nous interroger sur les conditions d'exercice du métier les premières années, et les moyens de favoriser le processus d'accrochage professionnel des enseignants novices.

Il peut paraitre surprenant de constater qu'aujourd'hui encore, comme il y a 40 ans, les jeunes enseignants éprouvent un " choc » à l'entrée dans le métier. Faut-il en conclure à un échec des politiques de formation des enseignants? Nous reviendrons tout du moins sur les premières affectations qui, selon nous, devraient se faire dans des établissements dans lesquels les collectifs de travail sont soudés et disponibles pour accompagner les enseignants novices. Ces personnes-ressources «officieuses» (Baudrit, 2011) sont dépourvues de tout rôle évaluateur, et peuvent ainsi apporter un soutien psychologique au stagiaire, là où le tuteur intervient davantage sur un accompagnement lié aux apprentissages académiques. Les réponses des collectifs sont également d'autant plus pertinentes qu'elles s'appuient sur une connaissance approfondie des élèves, du contexte, des outils déjà éprouvés et des motifs partagés.

Par ailleurs, l'usage de ressources vidéo sur le travail en classe, comme observé de plus en plus fréquemment en formation initiale des enseignants (Gaudin \& Chaliès, 2012), semble pouvoir modifier les conditions de l'alternance. En présentant la complexité de la classe à l'université, la vidéo constitue un outil pertinent pour concilier la double temporalité à laquelle les stagiaires sont confrontés. En ce sens, elle permet l'analyse de l'activité de l'enseignant pour mieux comprendre le décrochage professionnel et lutter contre le décrochage scolaire.

Il est également intéressant de constater récemment, dans plusieurs centres universitaires de France, l'ouverture de parcours visant à former les enseignants au tutorat et à la formation d'étudiants et de stagiaires, ainsi qu'à l'accompagnement des enseignants novices, au travers de réflexions sur les métiers d'enseignant et de formateur. Cette formation de formateurs d'adultes contribue également à la sensibilisation des tuteurs et des jeunes enseignants, et par conséquent des collectifs de travail, aux difficultés rencontrées par les enseignants novices, à l'importance de l'accueil dans les établissements et à celle de leur reconnaissance. Elle offre enfin un cadre d'analyse permettant de faire évoluer les dispositifs locaux de repérage et d'accompagnement des étudiants stagiaires en difficulté.

\section{BIBLIOGRAPHIE}

Adé, D., Gal-Petitfaux, N., \& Serres, G. (2014). Evolution de l'entrée dans le métier et perspectives de formation : Une étude à partir des ressources exploitées par les jeunes enseignants d'EPS au cours de leur première année d'expérience professionnelle. 8e biennale internationale de l'ARIS "Temps, temporalités et intervention en EPS et en sport ", Genève. 
Alava, S. (2015). L'enseignant face aux difficultés de la classe : étude des processus de décrochage professionnel. Colloque International « Conditions enseignantes», Lyon, janvier.

Archambault, I. (2006). Continuité et discontinuité dans le développement de l'engagement scolaire chez les élèves du secondaire : une approche centrée sur la personne pour prédire le décrochage. Thèse de doctorat, Université de Montréal, non publiée.

Baudrit, A. (2011). Mentorat et tutorat dans la formation des enseignants. Bruxelles : De Boeck. Bautier, E., Terrail, J.-P., Branca, S., Bonnery, S., Bebi, A., \& Lesort, B. (2002). « Décrochage scolaire : génèse et logique des parcours ». Rapport de recherche pour la DPD/MEN.

Bautier, E. (2003). Décrochage scolaire. Genèse et logique des parcours, VEI-Enjeux, 132, 30-45.

Beijaard, D., Verloop, N., \& Vermunt, J. (2000). Teachers' perceptions of professional identity : an exploratory study from a personal knowledge perspective. Teaching and Teacher Education, 16, 749-764.

Blaya, C. (2010). Décrochages scolaires : l'école en difficulté. Bruxelles : De Boeck.

Bonnery, S (2003). Le décrochage scolaire de l'intérieur : interaction de processus sociaux, cognitifs, subjectifs et langagiers. Les Sciences de l'éducation-Pour l'Ère nouvelle, 36, 39-58.

Bruno, F., Méard, J., \& Walter, E. (2013). Les dispositifs français de lutte contre le décrochage scolaire en collège : ce qui est prescrit et ce qui est mis en œuvre. L'orientation scolaire et professionnelle, 42(4), 439 - 459.

Clot, Y. (1995). Le travail sans l'homme? Paris : La Découverte.

Clot, Y. (1999). La fonction psychologique du travail. Paris : PUF.

Clot, Y. (2008). Travail et pouvoir d'agir. Paris : PUF.

Daniellou, F. (2002). Le travail des prescriptions. In Actes du $37^{\text {ième }}$ Congrès de la SELF « Nouvelles formes de travail, nouvelles formes d'analyse ", Aix-en-Provence, 9-16.

De Stercke, J., De Lièvre, B., Temperman, G., Cambier, J.-B., Renson, J.-M., Beckers, J., Leemans, M., \& Marechal, C. (2010). Difficultés d'insertion professionnelle dans l'enseignement secondaire ordinaire en Belgique francophone, Éducation \& Formation, e-294, 137-148.

Felix, C., Saujat, F., \& Combes, C. (2012). Des élèves en difficulté aux dispositifs d'aide : une nouvelle organisation du travail enseignant? Recherches en Education, 4 (HS), 19-30.

Feyfant, A. (2012). « Enseignement primaire : les élèves à risque (de décrochage) », Dossier d'actualité Veille et Analyses IFÉ, 80, Lyon, ENS de Lyon.

Flavier, E., \& Moussay, S. (2014). Répondre au décrochage scolaire. Expériences de terrain. Louvain-laNeuve : De Boeck.

Fortin, L., Marcotte, D., Diallo, T., Potvin, P., \& Royer, E. (2013). A multidimensional model of school dropout from an 8-year longitudinal study in a general high school population. European Journal of Psychology of Education, 28 (2), 563-583.

Gaudin, C. \& Chaliès, S. (2012). L'utilisation de la vidéo dans la formation professionnelle des enseignants novices. Revue Française de Pédagogie, 178, 115-130.

Gernet, I., \& Dejours, C. (2009). Evaluation du travail et reconnaissance. Nouvelle revue de psychologie, 8, 27-36.

Giust-Despraires, F. (1996). L'identité comme processus, entre liaison et déliaison. Éducation Permanente, 128, 63-70. 
Goigoux, R. (2002). L'évolution de la prescription adressée aux instituteurs : l'exemple de l'enseignement de la lecture entre 1972 et 2002. In Actes du 37ième Congrès de la SELF « Nouvelles formes de travail, nouvelles formes d'analyse ", Aix-en-Provence, 78-85.

Gu, Q., \& Day, C. (2007). Teachers resilience : A necessary condition for effectiveness. Teaching and Teacher Education, 23, 1302-1316.

Hernandez, P., \& Méard, J. (2014). Développer le collectif de travail pour lutter contre le décrochage professionnel : le cas des cadres d'éducation dans l'enseignement privé. Questions Vives - Recherches en Education, 21.

Hugon, M.-A. (2010). Lutter contre le décrochage scolaire : quelques pistes pédagogiques. Informations sociales, 161, 36-45.

Janosz, M. (2000). L'abandon scolaire chez les adolescents : perspective Nord-Américaine. VEIEnjeux, 122, 105-127.

Karsenti, T., \& Collin, S. (2009). L'autre décrochage scolaire. Formation et Profession, 16, 2-6.

Karsenti, T., Collin, S., \& Dumouchel, G. (2013). Le décrochage enseignant : état des connaissances. International Review of Education, 59(5), 549-568.

Leblanc, S., Ria, L., Dieumegard, G., Serres, G., \& Durand, M. (2008). Concevoir des dispositifs de formation professionnelle des enseignants à partir de l'analyse de l'activité dans une approche enactive. @ctivités, 5(1), 58-78.

Lee, V. E., \& Burkam, D. T. (2003). Dropping out of high school : The role of school organization and structure. American Educational Research Journal, 40(2), 353-393.

Leontiev, A. (1976). Le développement du psychisme. Paris : Editions sociales.

Lortie, D. (1975). Schoolteacher : A sociological study. Chicago : University of Chicago Press.

Martineau, S., \& Presseau, A. (2003). Le sentiment d'incompétence pédagogique des enseignants en début de carrière et le soutien à l'insertion professionnelle. Brock Éducation, 12(2), 54-67.

Méard, J. (2013). Les équipes de collèges qui luttent contre le décrochage scolaire ne font pas ce qu'on leur dit de faire. In Actes du Colloque International « Du décrochage scolaire à la réussite : le rôle des alliances éducatives, expériences européennes et nord-américaines », Université de Nice.

Millet, M., \& Thin, D. (2005). Ruptures scolaires. Paris : PUF.

Mukamurera, J., Martineau, S., Bouthiette, M., \& Ndoreraho, J.-P. (2012). Les programmes d'insertion professionnelle des enseignants dans les commissions scolaires du Québec : portrait et appréciation des acteurs. Éducation \& Formation, e-299, 13-35.

Moussay, S. (2014). Le double décrochage au cœur des échanges sur l'activité en classe. Dans E. Flavier \& S. Moussay (dir.), Répondre au décrochage scolaire - Expériences de terrain, 145-154. Louvain-la-Neuve : De Boeck.

Ria, L. (2009). De l'analyse de l'activité des enseignants en milieu difficile à la conception de dispositifs de formation. Dans M. Durand \& L. Filliettaz (Eds.), La place du travail dans la formation des adultes, 217-243. Paris : PUF.

Rots, I., Kelchtermans, G., \& Aelterman, A. (2012). Learning (not) to become a teacher : A qualitative analysis of the job entrance issue. Teaching and teacher Education, 28, 1-10.

Saujat, F. (2004). Comment les enseignants débutants entrent dans le métier. Formation et pratiques d'enseignement en questions. Revue des HEP de Suisse romande et du Tessin, 1, 97-106. 
Saujat, F. (2007). Enseigner : un travail. In V. Dupriez \& G. Chapelle (Eds.). Enseigner, 179-188.

Paris : PUF.

Saujat, F. (2010). Travail, formation et développement des professionnels de l'éducation : voies de recherche en sciences de l'éducation. Note de Synthèse pour l'Habilitation à Diriger les Recherches, Université de Provence - Aix-Marseille 1 (non publiée).

Schwartz, Y. (2012). Le paradigme ergologique ou le métier de philosophe. Toulouse : Octarès.

Smith, T., \& Ingersoll, R. (2004). What are the effects of induction and mentoring on beginning teacher turnover. American Educational Research Journal, 41(3), 681-714.

Vygotski, L. S. (1978). Mind in society: The development of higher psychological processes. Cambridge : Harvard University Press.

Vygotski, L. S. (1994). Le problème de la conscience dans la psychologie du comportement (trad. F. Sève). Société française, 50, 35-47.

Vygotski, L. S. (2003). Conscience, inconscient, émotions. Paris : La Dispute.

Yvon, F. \& Clot, Y. (2003). Apprentissage et développement dans l'analyse du travail enseignant. Pratiques psychologiques, 1, 19-35.

Zimmermann, P., Méard, J., \& Flavier, E. (2012). Quand les enseignants lèvent le doigt : le façonnage de l'identité professionnelle enseignante. Les Carrefours de l'éducation, 34, 195-210.

Zimmermann, P., \& Méard, J. (2015). L'accrochage professionnel des enseignants novices face à des élèves en situation de décrochage scolaire. Education \& Formation, e-304.

\section{RÉSUMÉS}

La question du lien entre les processus d'accrochage/décrochage professionnel des enseignants et la réussite scolaire des élèves apparait comme étant saillante lorsqu'on s'intéresse aux enseignants novices. Confrontés à des élèves en difficultés scolaires, leur identité professionnelle est bouleversée, au point, pour nombre d'entre eux, de se désengager progressivement. Une approche qualitative ancrée sur les postulats et la méthodologie de la clinique de l'activité (Clot, 2008) menée avec dix enseignants novices du primaire, a permis de souligner l'importance du collectif, celle de la reconnaissance mutuelle enseignant - élèves et les effets contrastés de l'alternance dans la formation des enseignants. Nous discutons l'hypothèse d'un lien spiralaire entre les processus d'accrochage/décrochage professionnel et la réussite des élèves, en pointant les nécessités d'être efficient, de renormaliser les prescriptions et d'une reconnaissance réciproque permettant la co-construction (enseignant et élèves) de sens accordé à leur travail. Des perspectives permettent d'interroger les circonstances favorables à l'accrochage lors des premières affectations et les dispositifs de formation récents dans les centres universitaires pour favoriser l'accompagnement des enseignants novices.

The link between the professional integrating/dropping process of teachers and student achievement appears as salient when looking at new teachers. Faced with students' learning difficulties, their professional identity is upset, to the point for many of them to gradually to leave the profession. A qualitative approach anchored on the assumptions and methodology of clinical activity (Clot, 2008) conducted with ten novice teachers from primary school, has highlighted the importance of collective work that of mutual recognition teacher - students, and the contrasting effects of alternation in teacher training. We discuss the hypothesis of a link between the professional process of dropping out and student success, pointing the necessity to 
be efficient, to renormalize prescriptions and a mutual recognition for a co-constructed (teacher and student) meaning given to their work. Prospects allow to question the favorable circumstances to integrate starting the first assignments, and recent training devices in academic centers encourage support for novice teachers.

INDEX

Mots-clés : activité, engagement, accrochage décrochage professionnel, identité professionnelle, clinique de l'activité

Keywords : activity, commitment, professional reintegration, dropout, professional identity, clinical activity

\section{AUTEUR}

\section{PHILIPPE ZIMMERMANN}

LISEC EA 2310, Université de Strasbourg 